\title{
Trends of Anticholinergics and Antipsychotics Prescribing at Chainama Hills College Hospital, Lusaka-Zambia
}

\author{
Francisca T Bwalya ${ }^{1 *(D)}$, James Mwanza ${ }^{2}$ (D) Ravi Paul ${ }^{2}$ (iD) \\ ${ }^{1}$ Chainama Hills College Hospital, Tertiary psychiatry hospital, Lusaka, Zambia, ${ }^{2}$ The University of Zambia, School of Medicine, \\ Department of Psychiatry, Lusaka, Zambia.
}

*Corresponding author: francisca.tbwalya@yahoo.com

\section{Abstract}

To cite: Bwalya FT, Mwanza J, Ravi P,. Trends of Anticholinergics and Antipsychotics Prescribing at Chainama Hills College Hospital, LusakaZambia. JPRM 2021, 3(2): 24-31. doi: 10.21617/jprm2021.327

Introduction: Antipsychotics are the main pharmacological treatment for psychosis. Anticholinergic drugs are sometimes prescribed with antipsychotics to treat or as prophylaxis for extrapyramidal side effects. Antipsychotic treatment guidelines recommend that anticholinergics should not be prescribed indiscriminately as prophylaxis for extrapyramidal side effects to patients using antipsychotic drugs, but only when there is high risk or evidence of extrapyramidal side effects, as they can cause significant central and peripheral side effects which have a potential to affect treatment outcomes. The objective of the study was to assess the trends in the prescribing of antipsychotics and anticholinergics.

Methods: A cross sectional study was conducted at Chainama Hills College Hospital in Zambia. An openended questionnaire was administered to 26 prescribers and 311 files for patients were reviewed who had an antipsychotic or anticholinergic drug prescribed. The prescription pattern of patient files was compared with the National Institute for Health and Care Excellence guidelines as a gold standard.

Results: The antipsychotic distribution showed that $76.1 \%$ were prescribed a typical antipsychotic, $18.1 \%$ an atypical antipsychotic and 5.8\% were on both typical and atypical antipsychotic. $28.2 \%$ of the patients on antipsychotics were prescribed anticholinergics (Trihexyphenidyl). $46.2 \%$ of the prescribing clinicians stated that they prescribe anticholinergics when a patient develops extrapyramidal side effects rather than concurrently with antipsychotics or when a high dose of antipsychotics has been prescribed.

Conclusion: The trend in antipsychotic and anticholinergic prescribing in Lusaka-Zambia were not consistent with recommended guidelines. Majority of patients are on typical antipsychotics rather than atypical antipsychotics. Most patients were administered above optimal dose of antipsychotics though polypharmacy was solemnly practiced. Recommend that further studies to explore factors contributing to this trend are conducted.

Keywords: antipsychotics, anticholinergics, prescribing, adherence 


\section{INTRODUCTION}

Antipsychotic drugs are the fundamental pharmacologic treatment for psychosis [1]. The key pharmacologic property of antipsychotics is their ability to block dopamine D2 receptors. Substantial blockade of D2 receptors, more common with conventional antipsychotics, cause motor side effects that are called extrapyramidal symptoms (EPS) such as parkinsonism (tremor, akinesia, and rigidity), akathisia, dystonia, and tardive dyskinesia (TD), occurring acutely or during chronic treatment [2]. There are a recent class of antipsychotics referred to as "atypical antipsychotic". These are so called due to the "atypical" clinical properties of equal positive symptom antipsychotic actions as conventional antipsychotics, but low extrapyramidal symptoms compared to conventional antipsychotics [3]. Therefore, there should be less need for anticholinergic co-medication to be prescribed with the atypical [4].

Anticholinergic drugs are used in the treatment of EPS [5]. Anticholinergics could be used to either treat EPS or prevent EPS in individuals at risk when administering antipsychotics $[2,6,7]$. These can cause peripheral side - effects such as dry mouth and constipation, as well as central adverse effects such as cognitive impairment, worsening of tardive dyskinesia, and delirium $[8,9,10]$. The current treatment guidelines for schizophrenia generally do not recommend the prophylactic and chronic use of anticholinergic [11,12]. Though, the administration of Trihexyphenidyl or alternative anticholinergic with antipsychotic medication for prophylaxis against extrapyramidal side effects (EPS) is a practice seen in psychiatry clinical practice [4]. Anticholinergic drugs have been reported to cause cognitive dysfunction in healthy individuals $[13,14,15]$, and induce delirium and hallucinations in healthy individuals [16,17]. Schizophrenia has cognitive disability as one of its major features causing functional disability thus anything that causes cognitive impairment, like anticholinergics, should be judiciously prescribed because of its possible compounding effect. Further, Trihexyphenidyl is abused by both the patient and the community for its euphoric and anxiolytic effects $[18,19]$.

There is a lack of adherence to current guidelines in antipsychotic drug prescribing in different countries [20]. A study that evaluated the prescribing practices in psychiatric residential facilities found high levels of antipsychotic polypharmacy $(57.2 \%)$ which was related to the total antipsychotic daily dose and to anticholinergic use [21]. A significant number of clinicians can benefit from changes in their psychotropic prescription [22]. The objective of the study was to assess the trends in the prescribing of antipsychotics and anticholinergics at Chainama Hills College Hospital in Zambia.

\section{METHODS AND MATERIALS}

The study was a cross sectional study done at Chainama Hills College Hospital. The objective of the study was to assess the trends in the prescribing of antipsychotics and anticholinergics. The study involved getting information of prescribing patterns of antipsychotics and anticholinergic during the period of December 2018 to March 2019 using patient files, and the reasons for the prescribing patterns gathered by providing questionnaires to prescribing clinicians. All qualified mental health prescribers based at the hospital and had consented to participate were included in the study; and all patients on antipsychotics except those whose medication regimen documentation was not complete. The study reviewed 316 patient's files employing a simple random sampling and 26 prescribers. The Maudsley Prescribing Guidelines in Psychiatry-12th edition and the National Institute for Health and Care Excellence (NICE) 2014 guidelines, were used as the standard tool for comparison of adherence. Self-administered Questionnaire for clinicians.

Information including age, sex, diagnosis, antipsychotics patient is on, if patient is on anticholinergic and any reported extrapyramidal adverse effects, were collected from patient files for the period of December 2018 to March 2019, and entered into an information form. Codes were used for files and no names of patients recorded. The data was collected in February and March 2019. Then information was collected from clinicians using self-administered questionnaires. Data was analysed using the Statistical Software Package (SPSS version 21). Statistical tests were at $5 \%$ significance interval. Percentage was used to measure the demographics, diagnosis and antipsychotics provided. The Pearson's ChiSquared test was used for comparison between different variables of patient data.

\section{RESULTS}

Table 1 shows the sex distribution with a larger percentage of males $(66.8 \%)$ that were prescribed with antipsychotics compared to female (33.2\%). Majority of the patients on antipsychotics were 
Bwalya FT, Mwanza J, Ravi P,. Trends of Anticholinergics and Antipsychotics Prescribing at Chainama Hills College Hospital, Lusaka-Zambia

below the age of 30 years $(50.33 \%)$, a good number (31.04\%) between 30 to 49 years and only $18.63 \%$ were 50 years and above. A significant number $(18.6 \%)$ were below 20 years, an age

Table 1: Demographics of patients and clinicians at Chainama Hills College Hospital, Zambia (n=310) group at increased risk of extrapyramidal side effects (EPS), as well as those above 60 years $(9.8 \%)$.

\begin{tabular}{llll}
\hline Characteristic Variable & Frequency (N) & Percent (\%) \\
\hline Sex of the patients & & 207 & \\
& Males & 103 & 64.7 \\
& Females & 10 & 32.2 \\
& Missing & & 3.1 \\
\hline Age group & Below 20 & 57 & \\
& $20-24$ & 53 & 17.8 \\
$25-29$ & 44 & 16.6 \\
$30-34$ & 36 & 13.8 \\
& $35-39$ & 35 & 11.2 \\
$40-44$ & 24 & 10.9 \\
$45-49$ & 13 & 7.5 \\
$50-54$ & 7 & 4.1 \\
$55-59$ & 7 & 2.2 \\
$60>$ & 30 & 2.2 \\
& Missing & 14 & 9.4 \\
& & & 4.4 \\
\hline Sex of the clinicians & 14 & 52 \\
& Females & 12 & 48 \\
\hline
\end{tabular}

Prescribing patterns of antipsychotics and anticholinergic

In table 2 majority of the patients were on typical antipsychotics $(76.1 \%)$, only $18.1 \%$ were on atypical antipsychotics, and 5.8\% were on both typical and atypical. The recommended drug of choice for psychosis treatment and antipsychotic use in general are atypical antipsychotics. 28.2\% of patients being prescribed antipsychotics were concurrently on anticholinergic. 
Table 2: Antipsychotic prescribing pattern an d anticholinergic prescribed prevalence at Chainama Hills College Hospital, Zambia

\begin{tabular}{cll}
\hline Pattern of prescription & Frequency (N) & Percentage (\%) \\
\hline Pattern of antipsychotic prescription & & \\
Typical & 236 & 76.1 \\
Atypical & 56 & 18.1 \\
Both typical and atypical & 18 & 5.8 \\
Route of administration & & \\
Oral & 201 & 64.8 \\
Intravenous & 6 & 1.9 \\
Intramuscular & 20 & 6.5 \\
More than one route & 83 & 26.8 \\
Patients on anticholinergic & & \\
yes & 83 & 28.2 \\
No & 211 & 71.8 \\
\hline
\end{tabular}

In Table 3, about $64.5 \%$ of the patients' regimen did not adhere to the recommended guidelines for the administration of antipsychotics. The lack of adherence was highly due to the administration of high doses (above optimal dose) of antipsychotics for episode which constituted $81.5 \%$, followed by

Table 3: Adherence description of antipsychotics at Chainama Hills College Hospital

\begin{tabular}{|c|c|c|}
\hline Characteristic variable & $\begin{array}{l}\text { Frequency } \\
\text { (N) }\end{array}$ & $\begin{array}{l}\text { Percentage } \\
(\%\end{array}$ \\
\hline \multicolumn{3}{|l|}{ Adherence to antipsychotic prescribing guidelines } \\
\hline Yes & 110 & 35.5 \\
\hline No & 200 & 64.5 \\
\hline \multicolumn{3}{|l|}{ Adherence of females } \\
\hline No & 73 & 70.9 \\
\hline Yes & 30 & 29.1 \\
\hline \multicolumn{3}{|l|}{ Adherence of Males } \\
\hline No & 127 & 61.4 \\
\hline Yes & 80 & 38.6 \\
\hline \multicolumn{3}{|l|}{ Lack of adherence observed } \\
\hline Polypharmacy & 2 & 1.0 \\
\hline Above optimal dose & 163 & 81.5 \\
\hline Below optimal dose & 26 & 13 \\
\hline Polypharmacy and above optimal dose & 9 & 4.5 \\
\hline
\end{tabular}

When given four choices, $15.4 \%$ of the clinicians said that they prescribe anticholinergics every time start a patient on antipsychotic medication and another $15.4 \%$ of the clinicians said that they would prescribe anticholinergics when patient is on high dose antipsychotic medication. While
$13 \%$ on a below optimal dose for episode, polypharmacy was seen in only $1 \%$, and $4.5 \%$ had a combination of polypharmacy and above optimal dose. 


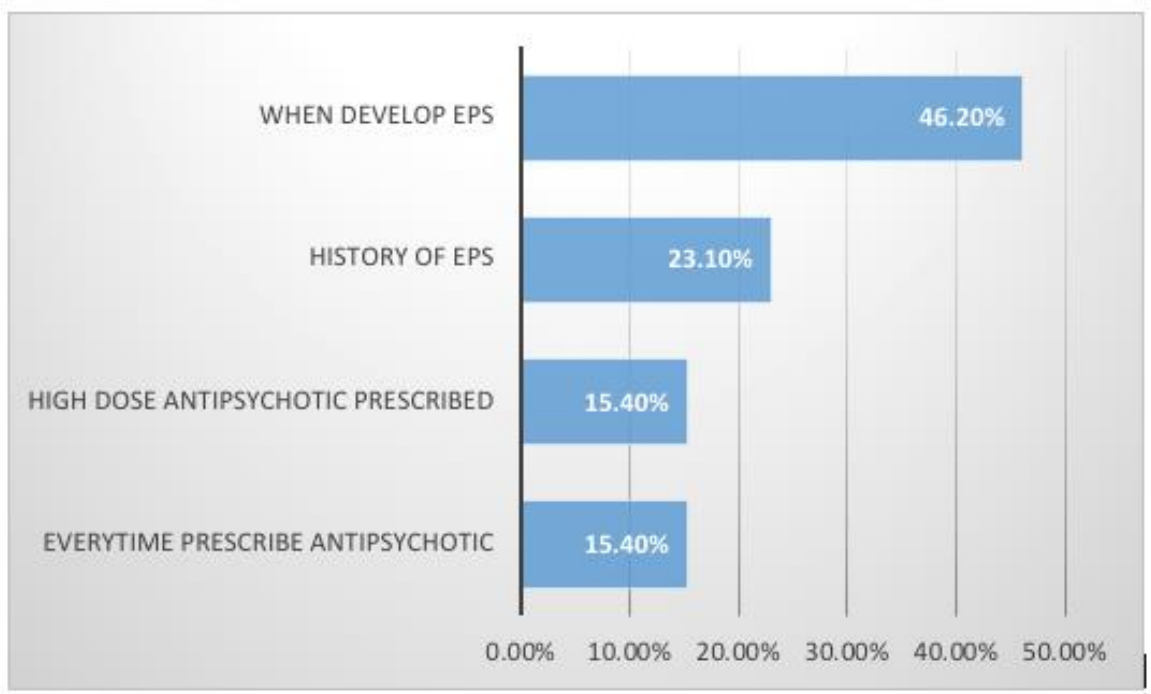

Figure 1: Clinician choice of when to prescribe an anticholinergic

\section{DISCUSSION}

We set out to assess the trends in the prescribing of antipsychotics and anticholinergics. The sex distribution showed that a larger percentage of patients that participated were male compared to female. This was a different picture compared to a United Kingdom study where the rates of prescribing were higher in females (incidence rate ratio (IRR) 1.092 (95\% CI 1.088 to 1.095). The same study showed that antipsychotic prescribing was higher in older people ( $80+$ vs 40-49; IRR 2.234 (2.222 to 2.246)) a great contrast to the current study which found that majority on antipsychotics were young, below the age of 30 years [23]. That could be an indication of the age distribution of the population or the preference of prescribing pattern of clinicians by age.

High typical antipsychotic prescribing is seen in the current study. There is an increasing tendency over the years to have a shift from prescribing of typical to atypical antipsychotics [24]. A study reported that for adults, second generation antipsychotic monotherapy prescriptions increased from $49 \%$ in 2006 to $71 \%$ in 2012, first-generation antipsychotic monotherapy prescriptions decreased from 29 to $14 \%$, and antipsychotic polypharmacy decreased from 23 to $15 \%$, respectively. Among the older adults, second-generation antipsychotic monotherapy prescriptions increased from 64 to $82 \%, \quad$ first - generation antipsychotic monotherapy prescriptions decreased from 29 to $12 \%$, and antipsychotic polypharmacy decreased from 7 to $6 \%$, respectively [24]. Compared to another study that was reviewed in Nigeria, [25] the findings of the current study in Zambia though still far from adherence levels to recommended standard guidelines in the prescribing of atypical antipsychotics as first line antipsychotic were found to be better. The findings of a study done in Nigeria reported that no patient was prescribed an atypical antipsychotic. The cost of atypical antipsychotic or its lack of supply in the hospitals could be causing this trend. Another study done in Nigeria that reported a low prescription rate for atypical antipsychotics explained that occurrence to be due to clinicians being aware that the large majority of patients in the country of study had a low income hence they were unwilling to prescribe them [26]. Other factors that could be contributing to the trend of high typical antipsychotic prescribing could be: the lack of knowledge of prescribing clinicians that atypical antipsychotics are the recommended first line drug of choice, lack of supply or availability of the atypical antipsychotics, lack of lobbying or guidance of policy makers/suppliers to consider the best treatment choice because they have not gotten that feedback.

A great proportion of the patients' regimen did not adhere to the recommended guidelines for the administration of antipsychotics. The lack of adherence being highly due to the administration of above optimal dose of antipsychotic for episode which was in greater than two-third while polypharmacy was minimal. These findings are similar to those shown by another study that compared the trends of antipsychotic prescribing of two years, 2005 and 2010. They found that the practice of prescribing high-dose antipsychotics and associated antipsychotic polypharmacy were common in 
both periods reviewed. The rates of high-dose antipsychotic prescription were $61.7 \%$ and $53.8 \%$ in 2005 and 2010, respectively. The rates of antipsychotic polypharmacy were $34.6 \%$ in 2005 and $34.0 \%$ in 2010 . The high-dose antipsychotics were significantly associated with antipsychotic polypharmacy [27]. Though there was a notable difference in the polypharmacy prevalence, the Zambian clinicians were not practicing polypharmacy much. In a European study a low level of polypharmacy was seen of $10.8 \%$ and the low trend towards polypharmacy was related to the nature of study population in the study, who were outpatients whose psychotic disorders may have been less severe [28]. The current study's low polypharmacy seen could be attributed to the fact that most of the patients recruited in the study were recently (less than four months) diagnosed patients. Thus, likely presenting with less severe and not chronic forms of psychotic disorders where a tendency for polypharmacy is seen. As well as a lack of availability of a variety of drugs to choose from could be contributing, unlike other places that have a range of medications to choose from.

Above a quarter of patients being prescribed antipsychotics were concurrently on anticholinergic, which is a significant portion of patients considering the side effects of anticholinergics; cognitive, peripheral and addiction which affect the quality of life of our patients. Considering that it is preventable and is against the standard recommended guidelines. Percentage in current study is better compared to study done in Asia that reported adjunctive anticholinergic prescribing to be at $56-75 \%$ [29]. Bahrain also showed a high prevalence of anticholinergic use which was $57.3 \%$ and $87.5 \%$ for outpatients on antipsychotic monotherapy and polytherapy, respectively [30]. A similar picture was seen in an African study with $62.2 \%$ of patients being on antipsychotic-anticholinergic combinations.

The current study showed a higher percentage use of Trihexyphenidyl, confirming the anecdotal experience reported in the country of study that mentioned that Trihexyphenidyl was the most commonly prescribed anticholinergic drug by the mental health professionals in Zambia [30]. What could be causing this trend, is it a cost factor or easy availability of the anticholinergic Trihexyphenidyl compared to other anticholinergics? It is still common practice $(82.6 \%)$ to observe that the common anticholinergic agent prescribed is
Trihexyphenidyl despite its increased risk for abuse [26]. About half of the prescribing clinicians, in the current study, stated that they prescribe anticholinergics when a patient develops EPS rather than concurrently with antipsychotics or when a high dose of antipsychotics has been prescribed. The response pattern of clinicians shows that majority, more than half, of the clinicians are making a choice of prescribing that is not in line with the recommended guidelines [31].

\section{CONCLUSION}

The trend in antipsychotic and anticholinergic prescribing in Lusaka-Zambia were not consistent with recommended guidelines. Majority of patients are administered typical antipsychotics rather than atypical antipsychotics. Most patients were administered above optimal dose of antipsychotics though polypharmacy was solemnly practiced. A significant number of patients were administered anticholinergics concurrently with antipsychotics against standard recommended guidelines. There will be need for various interventions, such as continuous medical education programs, to guide clinicians on the best practice in the prescribing of antipsychotics and anticholinergics.

DECLARATION

Acknowledgement Would like to acknowledge the input of Mr Christopher Njovu in the analysing of data. We also thank Dr Tony A Tsarkov, Dr Peter Petlovani and Dr Waqas Sheikh for their helpful comments. Further wish to thank Chainama Hills College Hospital Management and the staff for their warm reception and assistance.

Funding None

Competing interests There were no competing interests from all authors in this study.

\section{REFERENCES}

1. Miyake N, Miyamoto S, Jarskog LF. New serotonin/ dopamine antagonists for the treatment of schizophrenia. Clin. Schizophr. Relat. Psychoses 2012; 6: 122-133.

2. Miyamoto S, Merrill DB, Lieberman JA, Feischhacker WW, Marder SR. Antipsychotic drugs. In: Tasman A, Maj M, First M, Kay J, Lieberman J (eds). Psychiatry, 3rd edn. John Wiley and Sons, Chichester, 2008; 2161-2201.

3. Leucht S, Corves C, Arbter D, Engel RR, Li C, Davis JM. Second-generation versus firstgeneration antipsychotic drugs for schizophrenia: A meta-analysis. Lancet 2009; 373: 31-41.

4. Broekema W. J. ÆE I. W. de Groot $Æ$ P. N. van Harten. Simultaneous prescribing of atypical antipsychotics, conventional antipsychotics and 
anticholinergics-a European study. Published online: 27 February 2007

5. Bezchlibnyk-Butler KZ, Remington GJ. Antiparkinsonian drugs in the treatment of neuroleptic-induced extrapyramidal symptoms. Can. J. Psychiatry 1994; 39: 74-84.

6. Tune, L., Coyle, J.T. Acute extrapyramidal side effects: serum levels of neuroleptics and anticholinergics. Psychopharmacology (Berl.) $198175,9-15$.

7. Richelson, E. Receptor pharmacology of neuroleptics: relation to clinical effects. J. Clin. Psychiatry, 199960 (Suppl 10), 5-14.

8. MinzenbergMJ, PooleJH, BentonC, VinogradovS. Association of anticholinergic load with impairment of complex attention and memory in schizophrenia. Am. J. Psychiatry 2004; 161: 116-124.

9. Mintzer J, Burns A. Anticholinergic side-effects of drugs in elderly people. J. R. Soc. Med. 2000; 93: 457-462.

10. Strauss ME, Reynolds KS, Jayaram G, Tune LE. Effects of anticholinergic medication on memory in schizophrenia. Schizophr. Res. 1990; 3: 127129.

11. World Health Organization. Prophylactic use of anticholinergics in patients on long-term neuroleptic treatment. A consensus statement. World Health Organization heads of centres collaborating in WHO co-ordinated studies on biological aspects of mental illness. Br. J. Psychiatry 1990; 156: 412.

12. Lehman AF, Lieberman JA, Dixon LB et al. Practice guideline for the treatment of patients with schizophrenia, second edition. Am. J. Psychiatry 2004; 161: 1-56.

13. Ellis JR, Ellis KA, Bartholomeusz CF et al. Muscarinic and nicotinic receptors synergistically modulate working memory and attention in humans. Int. J. Neuropsychopharmacol. 2006; 9: 175-189.

14. McEvoy JP. A double-blind crossover comparison of antiparkinson drug therapy: Amantadine versus anticholinergics in 90 normal volunteers, with an emphasis on differentialeffectsonmemoryfunction. J. Clin. Psychiatry 1987; 48 (Suppl.): 20-23.

15. Drachman DA. Memory and cognitive function in man: Does the cholinergic system have a specific role? Neurology 1977; 27: 783-790.

16. Raedler TJ, Bymaster FP, Tandon R, Copolov D, Dean B. Towards a muscarinic hypothesis of schizophrenia. Mol. Psychiatry 2007; 12: 232246.

17. Perry EK, Perry RH. Acetylcholine and hallucinations: Disease-related compared to drug-induced alterations in human consciousness. Brain Cogn. 1995; 28: 240-258.

18. GP Pullen, NR Best, J Maguire. Anticholinergic drug abuse: a common problem? British medical journal (clinical research ed.) 289 (6445), 612, 1984
19. Neil Buhrich, Anthony Weller, Prue Kevans. Misuse of anticholinergic drugs by people with serious mental illness. Psychiatric services 51(7), 928-929, 2000

20. Rune A Kroken, Erik Johnsen, Torleif Ruud, Tore Wentzel-Larsen, Hugo A Jorgensen. Antipstchotics in Norwegian emergency wards, a cross-sectional national study. BMC psychiatry 9 (1), 24, 2009

21. L Ghio, W Natta, S Gotelli, L Ferrannini. Epidemiology and psychiatric sciences 20 (2), 171-179, 2011

22. Delbert $G$ Robinson, Nina R Schooler, Majnu John, Christoph U Corvell, Patricia Marcy, Jean Addington. Prescription practices in the treatment of first-episode schizophrenia spectrum disorders: data from the national RAISE-ETP study. American Journal of Psychiatry 172(3), 237-248, 2015

23. Louise Marston, Irwin Nazareth, Irene Petersen, Kate Walters, David PJ Osborn. Prescribing of antipsychotics in UK primary care: a cohort study. BMJ open 4 (12), 2014

24. Kenji Kochi, Izumi Sato, Chika Nishiyama, Sachiko Tanaka-Mizuno, Yuko Doi, Masaru Arai, Yosuke Fujii, Toshiyuki Matsunaga, Yusuke Ogawa, Toshi A Furukawa, Koji Kawakami. Trends in antipsychotic prescriptions for Japanese outpatients during 2006-2012: a descriptive epidemiological study. Pharmacoepidemiology and drug safety 26 (6), 642-656, 2017

25. AB Adeponle, AO Obembe, SO Adeyemi, GT Suleiman. Polypharmacy in psychiatric outpatient practice in northern Nigeria. African journal of psychiatry 10(4), 215-218, 2007

26. Imafidon $\mathrm{O}$ Agbonile, Oluwole Famuyiwa. Psychotropic drug prescribing in a Nigerian psychiatric hospital. International psychiatry 6(4), 96-98, 2009

27. Daeyoung Roh, Jhin-Goo Chang, Sol Yoon, Chan-Hyung Kim. Antipsychotic prescribing patterns in first-episode schizophrenia: a fiveyear comparison. Clinical Psychopharmacology and Neuroscience 13 (3), 275, 2015

28. Khalid A. J. Al Khaja1\#, Mohammed K. AlHaddad2, Reginald P. Sequeira1, Adel R. Antipsychotic and Anticholinergic Drug Prescribing Pattern in Psychiatry: Extent of Evidence-Based Practice in Bahrain*. Al-Offi3 Pharmacology \& Pharmacy, 2012, 3, 409-416 http://dx.doi.org/10.4236/pp.2012.34055

Published Online October 2012

29. Yoshio T, Uno J, Nakagawa M et al. Survey of the prescriptions for psychotherapy in Japanese inpatients with schizophrenia in 2006. Jpn. J. Clin. Psychopharmacol. 2010; 13: 1535-1545

30. Waqas Ahmed Sheikh. Prophylactic Use of Trihexyphenidyl (Artane) in Schizophrenia and Psychosis: A Critical Review of Literature to Guide for Evidence Based Practice in Zambia. Medical Journal of Zambia, Vol. 46 (2): 133 - 139 (2019) 
31. Mwanza J, Paul R, Ncheka JM, Petlovani P. Appropriateness of antipsychotic drugs prescribed for First episode psychosis by clinicians at Chainama Hills College Hospital in Lusaka. Health Press Zambia Bull. 2017; 1(4); (inclusive page numbers) 